\title{
QUANTIZED FEEDBACK CONTROL FOR NETWORKED CONTROL SYSTEMS UNDER INFORMATION LIMITATION
}

\author{
Qing-Quan Liu ${ }^{1,2}$, Guang-Hong Yang ${ }^{2,3}$ \\ ${ }^{1}$ School of Information Science and Engineering, Shenyang Ligong University \\ Shenyang, 110159, China \\ e-mail: lqqneu@163.com \\ ${ }^{2}$ College of Information Science and Engineering, Northeastern University \\ Shenyang, 110004, China \\ ${ }^{3}$ State Key Laboratory of Integrated Automation for Process Industries (Northeastern University) \\ Shenyang, 110004, China \\ crossref http://dx.doi.org/10.5755/j01.itc.40.3.630
}

\begin{abstract}
This paper investigates quantized control problems for linear time-invariant systems, where the sensors and controllers are geographically separated and connected by a digital communication network. This kind of problems arise in the source coding of signals between controllers and sensors in systems where feedback loops are closed using bandwidth-limited communication links. Sufficient conditions for stabilization of the unstable plant in the presence of limited information are derived. A lower bound on data rates, above which there exists a quantization, coding and control scheme to guarantee both stabilization and a certain control performance, is presented. The proof techniques rely on both information-theoretic and control-theoretic tools. An illustrative example is given to demonstrate the effectiveness of the proposed scheme.
\end{abstract}

Keywords: information limitation, networked control system, quantized control, feedback stabilization.

\section{Introduction}

In recent emerging applications, such as sensor networks, networked control systems and industrial control networks, the main purpose is to achieve some performance objectives, employing multiple sensors and actuators transmitting and receiving information over a digital communication network (see [24]). In a number of new technological settings, feedback control designs are constrained by bandwidth limitations in the communication channels between sensors, controller and actuators. This paper will discuss quantized control employing feedback channels with severe data-rate constraints.

Issues of the type discussed are motivated by several pieces of work in the recent literatures. The research on the interplay among coding, estimation, and control was initiated by [1]. A high-water mark in the study of quantized feedback using data-rate limited feedback channels is known as the data-rate theorem that states the larger the magnitude of the unstable poles, the larger the required data rate through the feedback loop. The intuitively appealing result was proved (see [2-5]), indicating that it quantifies a fundamental relationship between unstable physical systems and the rate at which information must be processed in order to stably control them. When the feedback channel capacity is near the data-rate limit, control designs typically exhibit chaotic instabilities. This result was generalized to different notions of stabilization and system models, and was also extended to multi-dimensional systems (see [6-8]). The research on Gaussian linear systems was addressed in [9-11]. Information theory was employed in control systems as a powerful conceptual aid, which extended existing fundamental limitations of feedback systems, and was used to derive necessary and sufficient conditions for robust stabilization of uncertain linear systems, Markov jump linear systems and unstructured uncertain systems (see [12-16]). The decentralized control schemes were addressed in [17]. The result on continuous-time linear Gaussian systems was derived in [18]. The result on time-varying communication channel was derived in [19]. The survey papers [20] and [21] gave a historical and technical account of the various formulations.

Existing analyses of quantized control under data-rate constraints rely on designing a quantization, coding and control policy to guarantee stability or stabilization of systems. Although the literatures above presented necessary and sufficient conditions for stability or stabilization, the cost must always become unbounded when the data-rate is reduced to the lower bound. This implies that the data rate which is too low affects performance significantly, regardless of the coding and control scheme in use. Thus, a natural issue is to minimize the amount of information needed to be transmitted while achieving a certain closed-loop performance. However, to date, very few 
works address the issue.

In this paper, we address quantized control designs to achieve both stabilization and a prescribed control performance in the presence of limited information. This is motivated by the fact that it is common in networked control systems to address the myriad standard control design issues such as rise time, gain and phase margins, etc. To deal with the issues, we need to have the capacity to transmit a great deal more data through the channel. A switched-adaptive quantization technique using $\mu$-law quantizers was addressed in [25]. Here, a quantization scheme for networked control systems is presented. Furthermore, we will generalize the technique for quantized state feedback to quantized output feedback. More specifically, we consider a single-input single-output (SISO) linear system with quantized measurements of the output.

The remainder of this paper is organized as follows: Section 2 introduces several preliminaries and problem statement. Section 3 addresses quantized control under information constraints. The results of numerical simulation are presented in Section 4. Conclusions are stated in Section 5.

\section{Preliminaries and problem statement}

Consider a linear control system described by

$$
\begin{aligned}
& X(t+1)=\mathbf{A} X(t)+\mathbf{B} U(t)+\mathbf{H} D(t), \\
& Y(t)=\mathbf{C} X(t)
\end{aligned}
$$

where $X(t)$ is the $\mathbb{R}^{n}$-valued plant state, $U(t)$ is the $\mathbb{R}^{m}$-valued control input, $Y(t)$ is the $\mathbb{R}^{p}$-valued observation output, $D(t)$ is the $\mathbb{R}^{q}$-valued bounded process disturbance, respectively. A, B, $\mathbf{H}$ and $\mathbf{C}$ are known constant matrices with appropriate dimensions (see Fig.l).

The following is assumed to hold:

$A_{0}$. Without loss of generality, suppose that the pair $(\mathbf{A}, \mathbf{B})$ is controllable, and the pair $(\mathbf{A}, \mathbf{C})$ is observable.

$A_{1}$. The sensors and controller are geographically separated and connected by noiseless communication channels. In this case, the measurement of the plant output is quantized but the control signal is not.

$A_{2}$. The initial condition $X(0)$ and disturbance $D(t)$ are possibly non-Gaussian and mutually independent random variables with zero mean, satisfying $\|X(0)\|^{2}<\phi_{0}<\infty$ and $\|D(t)\|^{2}<$ $\phi_{D}<\infty$, respectively.

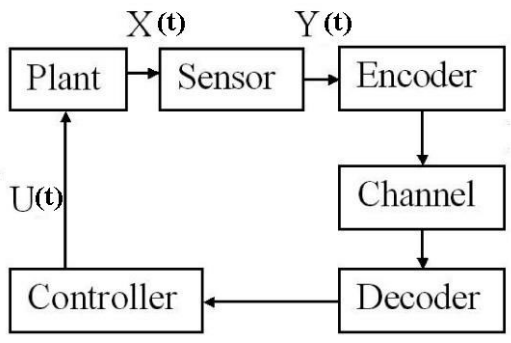

Figure 1. Networked control systems

As in [22], given a positive integer $N$ and a nonnegative real number $\Delta(t)$, we define the quantizer $q: \mathbb{R} \rightarrow \mathbb{Z}$ with sensitivity $\Delta(t)$ and saturation value $N$ by the formula

$$
q(z)= \begin{cases}N^{+}, & \text {if } z>(N+1 / 2) \Delta(t) \\ N^{-}, & \text {if } z \preceq-(N+1 / 2) \Delta(t) \\ \left\lfloor\frac{z}{\Delta(t)}+\frac{1}{2}\right\rfloor, & \text { if } z>-(N+1 / 2) \Delta(t) \\ & \text { and } z \preceq(N+1 / 2) \Delta(t)\end{cases}
$$

where we define $\lfloor z\rfloor:=\max \{k \in \mathbb{Z}:=k<$ $z, z \in \mathbb{R}\}$. The indices $N^{+}$and $N^{-}$will be employed when the quantizer saturates. The scheme to be used here is based on the hypothesis that it is possible to change the sensitivity (but not the saturation value) of the quantizer on the basis of available quantized measurements. The quantizer may counteract disturbances by switching repeatedly between "zooming out" and "zooming in" (see [22]). Assume that we have $n$ quantizers $q_{i}: \mathbb{R} \rightarrow \mathbb{Z}$ with sensitivity $\Delta_{i}(t)$ and saturation value $N_{i}(i=1, \cdots, n)$. Then we define the quantizer $q: \mathbb{R}^{n} \rightarrow \mathbb{Z}^{n}$ as

$$
q(X(t)):=\left[q_{1}\left(x_{1}(t)\right) q_{2}\left(x_{2}(t)\right) \cdots q_{n}\left(x_{n}(t)\right)\right]^{T} .
$$

We summarize the main definitions from [23]. Let $X$ and $\hat{X}$ denote two random variables. The differential entropy $h(X)$ is defined as

$$
h(X):=E_{X}\left[\log \frac{1}{p(X)}\right],
$$

where we denote by $p(X)$ the probability density function of $X$. Here, we write $\log _{2}(\cdot)$ simply as $\log (\cdot)$ throughout this paper. The conditional differential entropy of $X$ given $\hat{X}$ is defined as

$$
h(X \mid \hat{X}):=E_{X, \hat{X}}\left[\log \frac{1}{}\right.
$$


between $X$ and $\hat{X}$ is defined as

$$
I(X ; \hat{X}):=h(X)-h(X \mid \hat{X})=E_{X, \hat{X}}\left[\log \frac{p(X \mid \hat{X})}{p(X)}\right] .
$$

The information rate distortion function between $X$ and $\hat{X}$ is defined as

$$
R(D):=\inf _{p(\hat{X} \mid X) \in \Xi}\{I(X ; \hat{X})\}
$$

with $\Xi=\left\{p(\hat{X} \mid X): E_{X, \hat{X}}[d(X, \hat{X})] \preceq D\right\}$. Therein, $d(X, \hat{X})$ is a distortion function or distortion measure and $D$ is a constant given. The data rate $R(t)$ is given by

$$
R(t)=\frac{1}{h} I(X ; \hat{X})(\mathrm{bits} / \mathrm{s}),
$$

where $h$ is the transmission time for $I(X ; \hat{X})$. See [23] for further details.

Our performance objective is the linear quadratic (LQ) cost

$$
J:=\lim _{t \rightarrow \infty} \sup E X^{\prime}(t) \mathbf{Q} X(t),
$$

where $\mathbf{Q}$ is a positive definite matrix. Our purpose is to minimize the cost (3) in the presence of the process disturbance.

In this paper, we formulate quantized control problem for networked control systems with limited data rates of a digital communication channel. Then, we will deal with the LQ control problem for the fully- and partially-observed systems. Thus, the main problem proposed here is to present a lower bound on data rates, above which there exists a quantization, coding and control scheme to stabilize the system (1) in the mean square sense

$$
\lim _{t \rightarrow \infty} \sup E\|X(t)\|^{2}<\infty .
$$

\section{Digital control under information limitation}

This section deals with quantized feedback control for linear time-invariant systems under information limitation. First, a preliminary lemma from [23] is presented.

Lemma 1. Let $x \in \mathbb{R}$ denote a random variable and $\hat{x}$ denote an estimate of $x$. The expected distortion constraint is defined as $D \in \mathbb{R}^{+}$. For a given $D \succeq E(x-\hat{x})^{2}$, there must exist a quantization and coding scheme if $R(D)$ satisfies

$$
R(D) \succeq \frac{1}{2} \log \frac{\sigma^{2}(x)}{D}(\text { bits }),
$$

where $\sigma^{2}(x)=E(x-E x)^{2}$.
Proof. See [23].

Remark 1. Lemma 1 presents a lower bound on the amount of information which is needed to reconstruct the initial condition to some distortion fidelity. Then, the quantization and coding scheme is proposed to guarantee stabilization of the system (1) under the limitation of $R(D)$. We stress that the data rate $R(t)$ (bits/sample) of the communication channel must be greater than $R(D)$ such that there exists a control policy to stabilize the system (1). Clearly, more information available at the decoder will lead to better control performances.

In this case with information limitation, our main task is to present a lower bound on the data rate, above which there exists a quantization, coding and control scheme to achieve control objectives given. The data rate $R(t)$ plays a key role as we show below. First, we deal with quantized state feedback stabilization problem. Let $\lambda_{i}(\cdot)$ denote the $i$ th eigenvalue of a matrix and let $(\cdot)_{i j}$ denote an element of a matrix $(i, j=1, \cdots, n)$. Then, we have the following result:

Theorem 1. Consider the fully observed system (1). Let $\mu \in(0,1)$. Define

$$
\begin{aligned}
& \Phi:=\mu \boldsymbol{I}-(\boldsymbol{A}-\boldsymbol{B} \boldsymbol{K})^{\prime}(\boldsymbol{A}-\boldsymbol{B} \boldsymbol{K}), \\
& \Psi:=\boldsymbol{A}^{\prime} \boldsymbol{A}-(\boldsymbol{A}-\boldsymbol{B} \boldsymbol{K})^{\prime}(\boldsymbol{A}-\boldsymbol{B} \boldsymbol{K}) .
\end{aligned}
$$

Under assumptions $A_{0}-A_{2}$ stated in Section 2, there exists a control policy of the form

$$
U(t)=-\boldsymbol{K} \hat{X}(t)
$$

satisfying that all eigenvalues of $\boldsymbol{A}-\boldsymbol{B K}$ have magnitudes smaller than 1, such that the system (1) is stabilizable in the mean square sense (4) with

$$
J=\lim _{t-\infty} \sup X^{\prime}(t) \boldsymbol{Q} X(t) \preceq \frac{1}{1-\mu}\left\|\boldsymbol{H}^{\prime} \boldsymbol{Q} \boldsymbol{H}\right\| \phi_{D}
$$

if the data rate $R(t)$ satisfies the following inequality:

$$
R(t)>\frac{1}{2} \sum_{\left|\frac{\left(\boldsymbol{M} \Psi \boldsymbol{M}^{\prime}\right)_{i i}}{\lambda_{i}(\bar{\Phi})}\right|>1} \log \left|\frac{\left(\boldsymbol{M} \Psi \boldsymbol{M}^{\prime}\right)_{i i}}{\lambda_{i}(\bar{\Phi})}\right| \text { (bits/sample), }
$$

where $\boldsymbol{M}$ is the unitary matrix that diagonalizes

$$
\Phi=\boldsymbol{M}^{\prime} \bar{\Phi} \boldsymbol{M}
$$

Proof. The closed-loop system (1) may be written as

$$
X(t+1)=\mathbf{A} X(t)-\mathbf{B K} \hat{X}(t)+\mathbf{H} D(t) .
$$

Namely,

$X(t+1)=\mathbf{A}(X(t)-\hat{X}(t))+(\mathbf{A}-\mathbf{B K}) \hat{X}(t)+\mathbf{H} D(t)$. 
This implies that

$$
\begin{aligned}
E & X^{\prime}(t+1) \mathbf{Q} X(t+1) \\
= & \operatorname{trace}\left[\mathbf{Q} E X(t+1) X^{\prime}(t+1)\right] \\
= & \operatorname{trace}\left[\mathbf{Q A} E(X(t)-\hat{X}(t))(X(t)-\hat{X}(t))^{\prime} \mathbf{A}^{\prime}\right] \\
& \left.+\operatorname{trace}\left[\mathbf{Q H} E D(t) D^{\prime}(t) \mathbf{H}^{\prime}\right]\right) \\
& +\operatorname{trace}\left[\mathbf{Q}(\mathbf{A}-\mathbf{B K}) E \hat{X}(t) \hat{X}^{\prime}(t)(\mathbf{A}-\mathbf{B K})^{\prime}\right] \\
& +2 \operatorname{trace}\left[\mathbf{Q}(\mathbf{A}-\mathbf{B K}) E \hat{X}(t) D^{\prime}(t) \mathbf{H}^{\prime}\right] \\
& +2 \operatorname{trace}\left[\mathbf{Q A} E(X(t)-\hat{X}(t)) D^{\prime}(t) \mathbf{H}^{\prime}\right] \\
& +2 \operatorname{trace}\left[\mathbf{Q A} E(X(t)-\hat{X}(t)) \hat{X}^{\prime}(t)(\mathbf{A}-\mathbf{B K})^{\prime}\right]
\end{aligned}
$$

holds. By assumption $A_{2}$, we see that $X(t)$ and $D(t)$ are mutually independent. Then,

$$
\begin{gathered}
E \hat{X}(t) D^{\prime}(t)=0, \\
E(X(t)-\hat{X}(t)) D^{\prime}(t)=0 .
\end{gathered}
$$

Substituting (6) into (5), we may get

$$
\begin{array}{rl}
E X^{\prime}(t+1) \mathbf{Q} & X(t+1)=\operatorname{trace}\left[\mathbf{Q A} \Sigma_{X(t) \mid \hat{X}(t)} \mathbf{A}^{\prime}\right] \\
& +\operatorname{trace}\left[\mathbf{Q}(\mathbf{A}-\mathbf{B K}) \Sigma_{\hat{X}(t)}(\mathbf{A}-\mathbf{B K})^{\prime}\right] \\
& +\operatorname{trace}\left[\mathbf{Q H} \Sigma_{D(t)} \mathbf{H}^{\prime}\right]
\end{array}
$$

where we define

$$
\begin{aligned}
& \Sigma_{\hat{X}(t)}:=E \hat{X}(t) \hat{X}^{\prime}(t), \\
& \Sigma_{X(t) \mid \hat{X}(t)}:=E(X(t)-\hat{X}(t))(X(t)-\hat{X}(t))^{\prime}, \\
& \Sigma_{D(t)}:=E D(t) D^{\prime}(t) .
\end{aligned}
$$

If assume that

$$
\begin{aligned}
E X^{\prime}(t) \mathbf{Q} X(t) & >\operatorname{trace}\left[\mathbf{Q A} \Sigma_{X(t) \mid \hat{X}(t)} \mathbf{A}^{\prime}\right] \\
& +\operatorname{trace}\left[\mathbf{Q}(\mathbf{A}-\mathbf{B K}) \Sigma_{\hat{X}(t)}(\mathbf{A}-\mathbf{B K})^{\prime}\right]
\end{aligned}
$$

which is equivalent to

$$
\begin{array}{r}
\mu E X^{\prime}(t) \mathbf{Q} X(t)=\operatorname{trace}\left[\mathbf{Q A} \Sigma_{X(t) \mid \hat{X}(t)} \mathbf{A}^{\prime}\right] \\
+\operatorname{trace}\left[\mathbf{Q}(\mathbf{A}-\mathbf{B K}) \Sigma_{\hat{X}(t)}(\mathbf{A}-\mathbf{B K})^{\prime}\right]
\end{array}
$$

with $\mu \in(0,1)$, we may obtain

$$
\begin{aligned}
E X^{\prime}(t+1) \mathbf{Q} X(t+1)= & \mu E X^{\prime}(t) \mathbf{Q} X(t) \\
& +E D^{\prime}(t) \mathbf{H}^{\prime} \mathbf{Q} \mathbf{H} D(t) .
\end{aligned}
$$

This implies

$$
\begin{aligned}
E X^{\prime}(t) \mathbf{Q} X(t) & =\mu^{t} E X^{\prime}(0) \mathbf{Q} X(0) \\
& +\sum_{i=0}^{t-i-1} \mu^{i} E D^{\prime}(i) \mathbf{H}^{\prime} \mathbf{Q H} D(i) \\
& \preceq \preceq \mu^{t}\|\mathbf{Q}\| \phi_{0}+\frac{1-\mu^{t}}{1-\mu}\left\|\mathbf{H}^{\prime} \mathbf{Q} \mathbf{H}\right\| \phi_{D} .
\end{aligned}
$$

Thus,

$$
J=\lim _{t \rightarrow \infty} \sup E X^{\prime}(t) \mathbf{Q} X(t) \preceq \frac{1}{1-\mu}\left\|\mathbf{H}^{\prime} \mathbf{Q H}\right\| \phi_{D} .
$$

Clearly, if we can design a quantization and coding scheme such that (7) holds, there exists a control policy of the form $U(t)=-\mathbf{K} \hat{X}(t)$ to stabilize the system (1) with the cost $J$ subject to (8).

To guarantee that (7) holds, we set

$$
\begin{aligned}
\mu \operatorname{trace}\left[\Sigma_{X(t)}\right] & =\operatorname{trace}\left[\mathbf{A} \Sigma_{X(t) \mid \hat{X}(t)} \mathbf{A}^{\prime}\right] \\
& +\operatorname{trace}\left[(\mathbf{A}-\mathbf{B K}) \Sigma_{\hat{X}(t)}(\mathbf{A}-\mathbf{B K})^{\prime}\right] .
\end{aligned}
$$

Notice that

$$
\begin{aligned}
\Sigma_{\hat{X}(t)} & =E \hat{X}(t) \hat{X}^{\prime}(t)-E X(t) X^{\prime}(t)+E X(t) X^{\prime}(t) \\
& =\Sigma_{X(t)}-E\left(X(t) X^{\prime}(t)-\hat{X}(t) \hat{X}^{\prime}(t)\right) \\
& =\Sigma_{X(t)}-E(X(t)-\hat{X}(t))(X(t)-\hat{X}(t))^{\prime} \\
& =\Sigma_{X(t)}-\Sigma_{X(t) \mid \hat{X}(t)},
\end{aligned}
$$

where we define $\Sigma_{X(t)}:=E X(t) X^{\prime}(t)$. Substituting the equations above into (7), we have

$$
\begin{aligned}
\mu & \operatorname{trace}\left[\Sigma_{X(t)}\right] \\
= & \operatorname{trace}\left[\mathbf{A} \Sigma_{X(t) \mid \hat{X}(t)} \mathbf{A}^{\prime}\right] \\
& +\operatorname{trace}\left[(\mathbf{A}-\mathbf{B K}) \Sigma_{\hat{X}(t)}(\mathbf{A}-\mathbf{B K})^{\prime}\right] \\
= & \operatorname{trace}\left[\mathbf{A}^{\prime} \mathbf{A} \Sigma_{X(t) \mid \hat{X}(t)}\right] \\
& +\operatorname{trace}\left[(\mathbf{A}-\mathbf{B K})^{\prime}(\mathbf{A}-\mathbf{B K}) \Sigma_{\hat{X}(t)}\right] \\
= & \operatorname{trace}\left[\mathbf{A}^{\prime} \mathbf{A} \Sigma_{X(t) \mid \hat{X}(t)}\right] \\
& +\operatorname{trace}\left[(\mathbf{A}-\mathbf{B K})^{\prime}(\mathbf{A}-\mathbf{B K})\left(\Sigma_{X(t)}-\Sigma_{X(t) \mid \hat{X}(t)}\right)\right] \\
= & \operatorname{trace}\left[\left(\mathbf{A}^{\prime} \mathbf{A}-(\mathbf{A}-\mathbf{B K})^{\prime}(\mathbf{A}-\mathbf{B K})\right) \Sigma_{X(t) \mid \hat{X}(t)}\right] \\
& +\operatorname{trace}\left[(\mathbf{A}-\mathbf{B K})^{\prime}(\mathbf{A}-\mathbf{B K}) \Sigma_{X(t)}\right] . \\
&
\end{aligned}
$$

Thus, we have

$$
\operatorname{trace}\left(\Phi \Sigma_{X(t)}\right)=\operatorname{trace}\left(\Psi \Sigma_{X(t) \mid \hat{X}(t)}\right),
$$

where we define

$$
\begin{aligned}
& \Phi:=\mu \mathbf{I}-(\mathbf{A}-\mathbf{B K})^{\prime}(\mathbf{A}-\mathbf{B K}) \\
& \Psi:=\mathbf{A}^{\prime} \mathbf{A}-(\mathbf{A}-\mathbf{B K})^{\prime}(\mathbf{A}-\mathbf{B K}) .
\end{aligned}
$$

Then, it holds that

$$
E X^{\prime}(t) \Phi X(t)=E(X(t)-\hat{X}(t))^{\prime} \Psi(X(t)-\hat{X}(t)) .
$$

Let $\mathbf{M}$ be the unitary matrix that diagonalizes

$$
\Phi=\mathbf{M}^{\prime} \bar{\Phi} \mathbf{M}
$$

Define

$$
\begin{aligned}
\bar{X}(t) & :=\mathbf{M} X(t), \\
\tilde{X}(t) & :=\mathbf{M} \hat{X}(t) .
\end{aligned}
$$

It follows that

$$
E \bar{X}^{\prime}(t) \bar{\Phi} \bar{X}(t)=E(\bar{X}(t)-\tilde{X}(t))^{\prime} \mathbf{M} \Psi \mathbf{M}^{\prime}(\bar{X}(t)-\tilde{X}(t)) .
$$


Here, we quantize, encode $\bar{X}(t)$ and obtain the quantization value $\tilde{X}(t)$. Thus, it follows that

$\sum_{i=1}^{n} \lambda_{i}(\bar{\Phi}) E \bar{x}_{i}^{2}(t)=\sum_{i=1}^{n}\left(\mathbf{M} \Psi \mathbf{M}^{\prime}\right)_{i i} E\left(\bar{x}_{i}(t)-\tilde{x}_{i}(t)\right)^{2}$

Then, we set

$$
\Delta_{i}(t)=\sqrt{12\left|\frac{\lambda_{i}(\bar{\Phi})}{\left(\mathbf{M} \Psi \mathbf{M}^{\prime}\right)_{i i}}\right| E \bar{x}_{i}^{2}(t)}
$$

and

$$
N_{i}>\sqrt{\left|\frac{\left(\mathbf{M} \Psi \mathbf{M}^{\prime}\right)_{i i}}{\lambda_{i}(\bar{\Phi})}\right|}
$$

when

$$
\left|\frac{\left(\mathbf{M} \Psi \mathbf{M}^{\prime}\right)_{i i}}{\lambda_{i}(\bar{\Phi})}\right|>1
$$

In the case where $\left|\frac{\left(\mathbf{M} \Psi \mathbf{M}^{\prime}\right)_{i i}}{\lambda_{i}(\bar{\Phi})}\right| \preceq 1$, the corresponding $\bar{x}_{i}(t)$ needs not be quantized. Namely, we implement the quantizer of the form (2) with sensitivity $\Delta_{i}(t)$ and saturation value $N_{i}(\mathrm{i}=1, \cdots, \mathrm{n})$ subject to the conditions (9) and (10), respectively.

Now, we further argue the relationship between the data rate $R(t)$ and the control objectives given. We set $D_{i}(i=1, \cdots, n)$ subject to

$$
D_{i}=\left\{\begin{array}{cc}
\left|\frac{\lambda_{i}(\bar{\Phi})}{\left(\mathbf{M} \Psi \mathbf{M}^{\prime}\right)_{i i}}\right| E \bar{x}_{i}^{2}(t) & \text { when }\left|\frac{\left(\mathbf{M} \Psi \mathbf{M}^{\prime}\right)_{i i}}{\lambda_{i}(\bar{\Phi})}\right|>1 \\
E \bar{x}_{i}^{2}(t) & \text { when }\left|\frac{\left(\mathbf{M} \Psi \mathbf{M}^{\prime}\right)_{i i}}{\lambda_{i}(\Phi)}\right| \preceq 1
\end{array}\right.
$$

Then, by Lemma 1, we set

$$
R(D) \succeq \frac{1}{2} \log \frac{E \bar{x}_{i}^{2}(t)}{D_{i}} \succeq \frac{1}{2} \log \frac{\sigma^{2}\left(\bar{x}_{i}(t)\right)}{D_{i}} \text { (bits). }
$$

For transmitting the information of $\bar{x}_{i}(t)$, the corresponding data rate $R_{i}(t)$ must satisfy the following condition:

$$
\begin{aligned}
R_{i}(t)>\frac{1}{2} \log \left|\frac{\left(\mathbf{M} \Psi \mathbf{M}^{\prime}\right)_{i i}}{\lambda_{i}(\bar{\Phi})}\right| \text { (bits/sample) } \\
\text { when }\left|\frac{\left(\mathbf{M} \Psi \mathbf{M}^{\prime}\right)_{i i}}{\lambda_{i}(\bar{\Phi})}\right|>1
\end{aligned}
$$

or

$$
R_{i}(t)=0 \text { (bit/sample) when }\left|\frac{\left(\mathbf{M} \Psi \mathbf{M}^{\prime}\right)_{i i}}{\lambda_{i}(\bar{\Phi})}\right| \preceq 1 \text {. }
$$

Thus, it follows that

$$
\begin{aligned}
R(t) & >\sum_{i=1}^{n} R_{i}(t) \\
& =\frac{1}{2} \sum_{\left|\frac{\left(\mathbf{M} \Psi \mathbf{M}^{\prime}\right)_{i i}}{\lambda_{i}(\bar{\Phi})}\right|>1} \log \left|\frac{\left(\mathbf{M} \Psi \mathbf{M}^{\prime}\right)_{i i}}{\lambda_{i}(\bar{\Phi})}\right| \text { (bits/sample). }
\end{aligned}
$$

Remark 2. In Theorem 1, the parameter $\mu$ plays a key role in the relationship between control performances and the data rate of the communication channel. Namely, if we want to obtain better performance, we have to increase the data rate.

We now turn to the problem of stabilizing the system

$$
\begin{aligned}
& X(t+1)=\mathbf{A} X(t)+\mathbf{B} u(t)+\mathbf{H} D(t) \\
& y(t)=\mathbf{C} X(t)
\end{aligned}
$$

with quantized measurements of the output. Here, $u(t) \in \mathbb{R}$ is the control input and $y(t) \in \mathbb{R}$ is the plant output. The case in which the plant output as well as the control input is a scalar is the most interesting. Clearly, considering this case avoids extraneous complexity and makes our conclusions most transparent.

Then, we establish the following result:

Theorem 2. Consider the partially-observed system (11). Let $\mu \in(0,1)$. Denote by $\hat{y}(t)$ the quantization value of $y(t)$. Define

$$
\begin{aligned}
& \Xi:=\mu \boldsymbol{I}-(\boldsymbol{A}-\boldsymbol{B} k \boldsymbol{C})^{\prime}(\boldsymbol{A}-\boldsymbol{B} k \boldsymbol{C}), \\
& \Omega:=\boldsymbol{A}^{\prime} \boldsymbol{A}-(\boldsymbol{A}-\boldsymbol{B} k \boldsymbol{C})^{\prime}(\boldsymbol{A}-\boldsymbol{B} k \boldsymbol{C}) .
\end{aligned}
$$

Under assumptions $A_{0}-A_{2}$ from Section 2, there exists a control policy of the form

$$
u(t)=-k \hat{y}(t)
$$

satisfying that all eigenvalues of $\boldsymbol{A}-\boldsymbol{B} k \boldsymbol{C}$ have magnitudes smaller than 1, such that the system (11) is stabilizable in the mean square sense (4) with

$$
J=\lim _{t-\infty} \sup X^{\prime}(t) \boldsymbol{Q} X(t) \preceq \frac{1}{1-\mu}\left\|\boldsymbol{H}^{\prime} \boldsymbol{Q} \boldsymbol{H}\right\| \phi_{D}
$$

if the data rate $R(t)$ satisfies the following inequality:

$$
R(t)>\frac{1}{2} \sum_{\left|\frac{\left(N \Omega N^{\prime}\right)_{i i}}{\lambda_{i}(\Xi)}\right|>1} \log \left|\frac{\left(N \Omega N^{\prime}\right)_{i i}}{\lambda_{i}(\Xi)}\right|(\text { bits/sample })
$$

where $\mathrm{N}$ is the unitary matrix that diagonalizes

$$
\Xi=N^{\prime} \bar{\Xi} N
$$

Proof. The closed-loop system (11) may be written as

$$
X(t+1)=\mathbf{A} X(t)-\mathbf{B} k \mathbf{C} \hat{X}(t)+\mathbf{H} D(t) .
$$

Namely,

$X(t+1)=\mathbf{A}(X(t)-\hat{X}(t))+(\mathbf{A}-\mathbf{B} k \mathbf{C}) \hat{X}(t)+\mathbf{H} D(t)$. 
Arguing as before, if we assume that

$$
\begin{aligned}
E X^{\prime}(t) & \mathbf{Q} X(t)>\operatorname{trace}\left[\mathbf{Q A} \Sigma_{X(t) \mid \hat{X}(t)} \mathbf{A}^{\prime}\right] \\
& +\operatorname{trace}\left[\mathbf{Q}(\mathbf{A}-\mathbf{B} k \mathbf{C}) \Sigma_{\hat{X}(t)}(\mathbf{A}-\mathbf{B} k \mathbf{C})^{\prime}\right]
\end{aligned}
$$

which is equivalent to

$$
\begin{gathered}
\mu E X^{\prime}(t) \mathbf{Q} X(t)=\operatorname{trace}\left[\mathbf{Q A} \Sigma_{X(t) \mid \hat{X}(t)} \mathbf{A}^{\prime}\right] \\
+\operatorname{trace}\left[\mathbf{Q}(\mathbf{A}-\mathbf{B} k \mathbf{C}) \Sigma_{\hat{X}(t)}(\mathbf{A}-\mathbf{B} k \mathbf{C})^{\prime}\right]
\end{gathered}
$$

with $\mu \in(0,1)$, we may obtain

$$
\begin{aligned}
E X^{\prime}(t+1) \mathbf{Q} X(t+1)= & \mu E X^{\prime}(t) \mathbf{Q} X(t) \\
& +E D^{\prime}(t) \mathbf{H}^{\prime} \mathbf{Q H} D(t) .
\end{aligned}
$$

This implies

$$
\begin{aligned}
E X^{\prime}(t) \mathbf{Q} X(t) & =\mu^{t} E X^{\prime}(0) \mathbf{Q} X(0) \\
& +\sum_{i=0}^{t-i-1} \mu^{i} E D^{\prime}(i) \mathbf{H}^{\prime} \mathbf{Q H} D(i) \\
& \preceq \mu^{t}\|\mathbf{Q}\| \phi_{0}+\frac{1-\mu^{t}}{1-\mu}\left\|\mathbf{H}^{\prime} \mathbf{Q} \mathbf{H}\right\| \phi_{D} .
\end{aligned}
$$

Thus, it holds that

$$
J=\lim _{t \rightarrow \infty} \sup E X^{\prime}(t) \mathbf{Q} X(t) \preceq \frac{1}{1-\mu}\left\|\mathbf{H}^{\prime} \mathbf{Q H}\right\| \phi_{D} .
$$

Clearly, if we can design a quantization and coding scheme such that (12) holds, there exists a control policy of the form $u(t)=-k \hat{y}(t)$ to stabilize the system (11) with the cost $J$ subject to (13).

To guarantee that (12) holds, we set

$$
\begin{aligned}
\mu \operatorname{trace}\left[\Sigma_{X(t)}\right] & =\operatorname{trace}\left[\mathbf{A} \Sigma_{X(t) \mid \hat{X}(t)} \mathbf{A}^{\prime}\right] \\
& +\operatorname{trace}\left[(\mathbf{A}-\mathbf{B} k \mathbf{C}) \Sigma_{\hat{X}(t)}(\mathbf{A}-\mathbf{B} k \mathbf{C})^{\prime}\right] .
\end{aligned}
$$

Thus, it follows that

$$
\operatorname{trace}\left(\Xi \Sigma_{X(t)}\right)=\operatorname{trace}\left(\Omega \Sigma_{X(t) \mid \hat{X}(t)}\right)
$$

where

$$
\begin{aligned}
& \Xi:=\mu \mathbf{I}-(\mathbf{A}-\mathbf{B} k \mathbf{C})^{\prime}(\mathbf{A}-\mathbf{B} k \mathbf{C}), \\
& \Omega:=\mathbf{A}^{\prime} \mathbf{A}-(\mathbf{A}-\mathbf{B} k \mathbf{C})^{\prime}(\mathbf{A}-\mathbf{B} k \mathbf{C}) .
\end{aligned}
$$

Then,

$$
E X^{\prime}(t) \Xi X(t)=E(X(t)-\hat{X}(t))^{\prime} \Omega(X(t)-\hat{X}(t)) .
$$

Let $\mathbf{N}$ be the unitary matrix that diagonalizes

$$
\Xi=\mathbf{N}^{\prime} \Xi \mathbf{N}
$$

Define

$$
\begin{aligned}
& \bar{X}(t):=\mathbf{N} X(t), \\
& \tilde{X}(t):=\mathbf{N} \hat{X}(t) .
\end{aligned}
$$

Thus,

$$
\begin{aligned}
E \bar{X}^{\prime}(t) & \bar{\Xi} \bar{X}(t) \\
& =E(\bar{X}(t)-\tilde{X}(t))^{\prime} \mathbf{N} \Omega \mathbf{N}^{\prime}(\bar{X}(t)-\tilde{X}(t)) .
\end{aligned}
$$

Here, $\tilde{X}(t)$ may be viewed as the estimate value of $\bar{X}(t)$. Thus, it follows that

$$
\sum_{i=1}^{n} \lambda_{i}(\bar{\Xi}) E \bar{x}_{i}^{2}(t)=\sum_{i=1}^{n}\left(\mathbf{N} \Omega \mathbf{N}^{\prime}\right)_{i i} E\left(\bar{x}_{i}(t)-\tilde{x}_{i}(t)\right)^{2} .
$$

It follows from the observability of the pair (A, C) that

$$
I(y(t) ; \hat{y}(t))=I(X(t) ; \hat{X}(t)) .
$$

Furthermore,

$$
I(X(t) ; \hat{X}(t))=I(\bar{X}(t) ; \tilde{X}(t)) .
$$

Then,

$$
\begin{aligned}
R(D) & =\inf _{p(\hat{y}(t) \mid y(t)) \in \Gamma^{\prime}}\{I(y(t) ; \hat{y}(t))\} \\
& =\inf _{p(\tilde{X}(t) \mid \bar{X}(t)) \in \Gamma}\{I(\bar{X}(t) ; \tilde{X}(t))\}
\end{aligned}
$$

where $\Gamma=\{p(\tilde{X}(t) \mid \bar{X}(t)): E[d(\bar{X}(t), \tilde{X}(t))] \preceq$ $D\}$.

Then, we set

$$
\Delta(t)=\sqrt{12 \Pi_{i \in \Lambda}\left|\frac{\lambda_{i}(\bar{\Xi})}{\left(\mathbf{N} \Omega \mathbf{N}^{\prime}\right)_{i i}}\right| E y^{2}(t)}
$$

and

$$
N>\sqrt{\prod_{i \in \Lambda}\left|\frac{\lambda_{i}(\overline{\bar{\Xi}})}{\left(\mathbf{N} \Omega \mathbf{N}^{\prime}\right)_{i i}}\right|}
$$

where $\Lambda=\left\{i \in\{1, \cdots, n\}:\left|\frac{\left(\mathbf{N} \Omega \mathbf{N}^{\prime}\right)_{i i}}{\lambda_{i}(\bar{\Xi})}\right|>1\right\}$.

Namely, we implement the quantizer of the form (2) with sensitivity $\Delta(t)$ and saturation value $N$ subject to the conditions (14) and (15), respectively.

Now, we further argue the relationship between the data rate $R(t)$ and the control objectives given. We set $D$ subject to

$$
D=\Pi_{i \in \Lambda}\left|\frac{\lambda_{i}(\bar{\Xi})}{\left(\mathbf{N} \Omega \mathbf{N}^{\prime}\right)_{i i}}\right| E y^{2}(t)
$$

For transmitting the information of $y(t)$, the corresponding data rate $R(t)$ must satisfy the following condition:

$$
R(t)>\frac{1}{2} \log \Pi_{i \in \Lambda}\left|\frac{\left(\mathbf{N} \Omega \mathbf{N}^{\prime}\right)_{i i}}{\lambda_{i}(\bar{\Xi})}\right| \text { (bits/sample). }
$$


Remark 3. Although the argument presented above only deals explicitly with the single-input singleoutput (SISO) case, it is possible to extend the result of Theorem 2 to deal with multi-input multi-output (MIMO) case.

\section{Numerical example}

Issues of the type discussed in this paper arise in the coordinated motion control of autonomous and semiautonomous mobile agents, e.g., unmanned air vehicles (UAVs), unmanned ground vehicles (UGVs), and unmanned underwater vehicles (UUVs). To illustrate the proposed quantization and control scheme for linear control systems under information limitation, we present a practical example, where three of the states of an unmanned air vehicle evolve in discrete-time according to

$$
\begin{gathered}
X(t+1)=\left[\begin{array}{ccc}
1.92 & 1.53 & -0.23 \\
0.26 & -2.37 & 0.61 \\
0.74 & 0.27 & 1.75
\end{array}\right] X(t) \\
+\left[\begin{array}{l}
2.12 \\
1.15 \\
0.24
\end{array}\right] u(t)+2.3 W(t), \\
y(t)=\left[\begin{array}{lll}
0.31 & -0.30 & 0.29
\end{array}\right] X(t)
\end{gathered}
$$

The initial condition $X(0)=\left[\begin{array}{lll}180 & 10 & -80\end{array}\right]^{\prime}$ and $\phi_{D}=0.51$. Let the sampling period $h=20 \mathrm{~ms}$.

We select the controller gain $k=2.24$ satisfying that all eigenvalues of $A-B k C$ lie inside the unit circle. If let $R(t)=150$ (bits/s) and $\mu=1$, a corresponding simulation is given in Fig. 2. Clearly, the closed-loop system is unstable, regardless of the quantization and control scheme in use, when the data rate $R(t)$ is smaller than the lower bound (i.e., 156 bits/s), which was derived in [3], [6], [9] and etc. To guarantee stability of the system above, we increase the data rate and set $\mu=1, R(t)=160$ (bits/s). The corresponding simulation is given in Fig. 3. Here we are interested in the qualitative dynamics of linear control systems operating near the lower bound. However, the cost $J$ is rather poor although the system may be stabilizable in the mean square sense.

To improve the control performance, we implement the quantization and control scheme on the basis of the condition proposed in Theorem 2. If let $\mu=0.64$ and $R(t)=400$ (bits/s), we may obtain $J=17.39$. The corresponding simulation is given in Fig. 4. Clearly, the larger data rate may lead to the better control performance. If we change the controller law, and set $k=2.57$, we may obtain $R(t)=460$ (bits/s) and $J=12.51$. The corresponding simulation is given in Fig. 5. It means that the control law

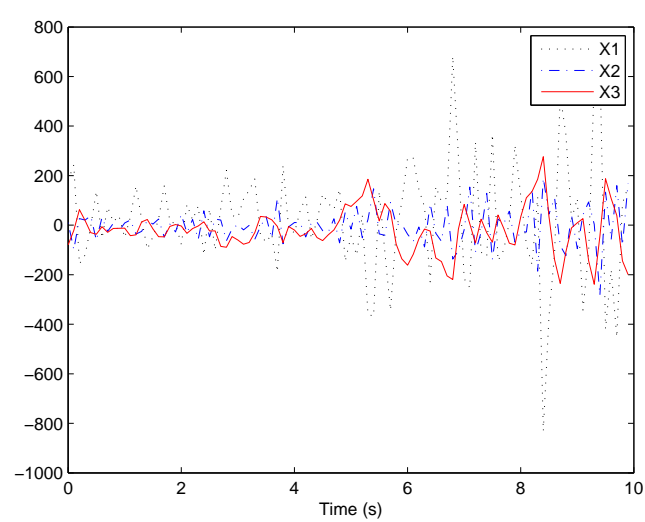

Figure 2. The system state responses when $\mu=1, k=2.24$.

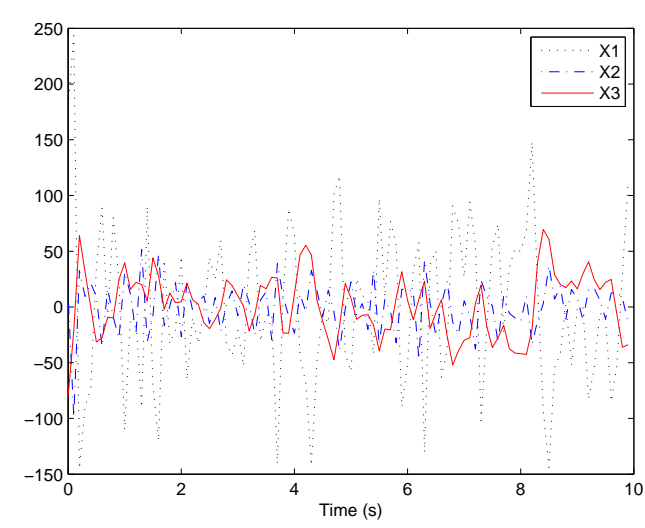

Figure 3. The system state responses when $\mu=1, k=2.24$.

has an important effect on the control performance in the case with information limitation.

\section{Conclusion}

This paper addressed quantized control problems for networked control systems with limited data rates. The approach taken here relied on both informationtheoretic and control-theoretic tools. We developed a number of techniques, for both fully- and partiallyobserved systems, which enable one to achieve some given control objectives. The simulation results have illustrated the effectiveness of the proposed quantization, coding and control scheme.

\section{Acknowledgment}

This work was supported in part by the Funds for Creative Research Groups of China (No. 60821063), National 973 Program of China (Grant No. 2009CB32 


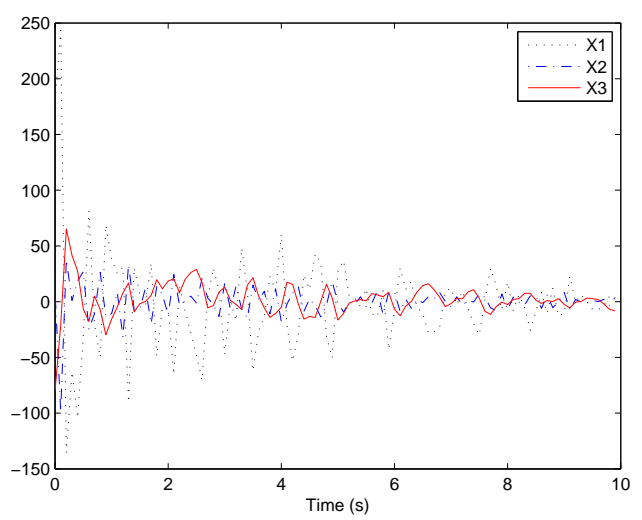

Figure 4. The system state responses when $\mu=0.64, k=2.24$.

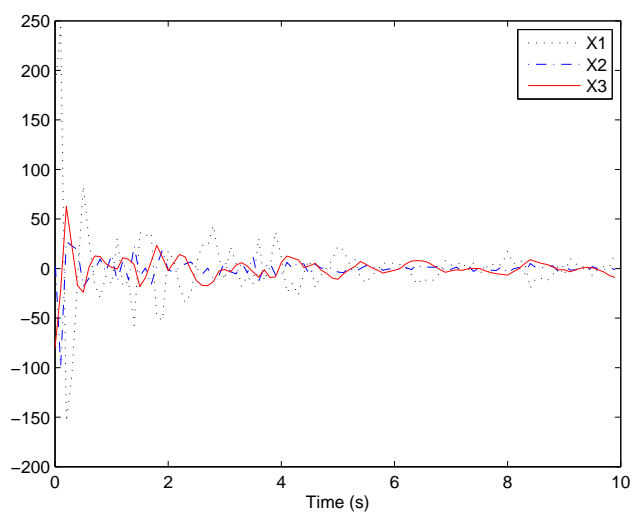

Figure 5. The system state responses when $\mu=0.64, k=2.57$.

0604), the Funds of National Science of China (Grant No. 60974043), the 111 Project (B08015), and the Funds of Doctoral Program of Ministry of Education, China (20100042110027).

\section{References}

[1] W.S. Wong, R.W. Brockett. Systems with finite communication bandwidth constraints II: Stabilization with limited information feedback, IEEE Transactions on Automatic Control, Vol.44, No.5, 1999, 1049-1053.

[2] J. Baillieul. Feedback designs for controlling device arrays with communication channel bandwidth constraints, in ARO Workshop on Smart Structures, Pennsylvania State Univ, Aug. 1999.

[3] J. Baillieul. Feedback designs in information based control, Stochastic Theory and Control Proceedings of a Workshop Held in Lawrence, Kansas, B. PasikDuncan, Ed. New York: Springer-Verlag, 2001, 35-57.
[4] J. Baillieul. Data-rate requirements for nonlinear feedback control, Proc. 6th IFAC Symp. Nonlinear Control Syst., Stuttgart, Germany, 2004, 1277-1282.

[5] K. Li, J. Baillieul. Robust quantization for digital finite communication bandwidth (DFCB) control, IEEE Transactions on Automatic Control, Vol.49, No.9, 2004, 1573-1584.

[6] G.N. Nair, R.J. Evans. Stabilizability of stochastic linear systems with finite feedback data rates, SIAM J. Control Optim., Vol.43, No.2, 2004, 413-436.

[7] N. Elia, S. Mitter. Stabilization of linear systems with limited information, IEEE Transactions on Automatic Control, Vol.46, No.9, 2001, 1384-1400.

[8] N. Elia. When Bode meets Shannon: Control-oriented feedback communication schemes, IEEE Transactions on Automatic Control, Vol.49, No.9, 2004, 1477 1488.

[9] S. Tatikonda, S. Mitter. Control under communication constraints, IEEE Transactions on Automatic Control, Vol.49, No.7, 2004, 1056-1068.

[10] S. Tatikonda, S. Mitter. Control over noisy channels, IEEE Transactions on Automatic Control, Vol.49, No.7, 2004, 1196-1201.

[11] S. Tatikonda, A. Sahai, S. Mitter. Stochastic linear control over a communication channel, IEEE Transactions on Automatic Control, Vol.49, No.9, 2004, 1549-1561.

[12] N.C. Martins, M.A. Dahleh, N. Elia. Feedback stabilization of uncertain systems in the presence of a direct link, IEEE Transactions on Automatic Control, Vol.51, No.3, 2006, 438-447.

[13] N.C. Martins, M.A. Dahleh. Feedback control in the presence of noisy channels: 'Bode-like' fundamental limitations of performance, IEEE Transactions on Automatic Control, Vol.53, No.7, 2008, 1604-1615.

[14] G.N. Nair, S. Dey, R.J. Evans. Infimum data rates for stabilizing Markov jump linear systems, Proc. IEEE Conf. Decision and Control, 2003, 1176-1181.

[15] A. Sahai, S. Mitter. The necessity and sufficiency of anytime capacity for stabilization of a linear system over a noisy communication link Part I: Scalar systems, IEEE Transactions on Automatic Control, Vol.52, No.8, 2006, 3369-3395.

[16] J.Q. Sun, S.M. Djouadi. Robust stabilization over communication channels in the presence of unstructured uncertainty, IEEE Transactions on Automatic Control, Vol.54, No.4, 2009, 830-834.

[17] S. Yüksel, T. Basar. Communication constraints for decentralized stabilizability with time-invariant policies, IEEE Transactions on Automatic Control, Vol.52, No.6, 2007, 1060-1066.

[18] C.D. Charalambous, A. Farhadi, S.Z. Denic. Control of continuous-time linear Gaussian systems over additive Gaussian wireless fading channels: A separation principle, IEEE Transactions on Automatic Control, Vol.53, No.4, 2008, 1013-1019.

[19] P. Minero, M. Franceschetti, S. Dey, G.N. Nair. Data rate theorem for stabilization over time-varying feedback channels, IEEE Transactions on Automatic 
Control, Vol.54, No.2, 2009, 243-255.

[20] J. Baillieul, P. Antsaklis. Control and communication challanges in networked real time systems, Proceedings of IEEE Special Iss. Emerg. Technol. Netw. Control Syst, USA: IEEE, 2007, 9-28.

[21] G. N. Nair, F. Fagnani, S. Zampieri, R.J. Evans. Feedback control under data rate constraints: An overview, Proceedings of IEEE Special Iss. Emerg. Technol. Netw. Control Syst, USA: IEEE, 2007, 108137.

[22] D. Liberzon, D. Nešićc. Input-to-state stabilization of linear systems with quantized state measurements, IEEE Transactions on Automatic Control, Vol.52, No.5, 2007, 767-781.

[23] T. Cover, J. Thomas. Elements of Information Theory. New York: Wiley, 2006.

[24] P. Orlowski. System degradation factor for networked control systems, Information Technology And Control, Vol.37, No.3, 2008, 233-244.

[25] Z. Peric, J. Nikolic. A. Mosic, S. Panic, A switchedadaptive quantization technique using $\mu$-law quantizers, Information Technology And Control, Vol.39, No.4, 2010, 317-320.

Received December 2010. 